\title{
Production of radiotoxic isotopes in LBE spallation targets: recent extensions of the INCL4 model and experimental validation
}

\author{
Th. Aoust ${ }^{1,2, a}$, J. Cugnon ${ }^{2}$, and J. Wagemans ${ }^{1}$ \\ 1 SCK•CEN, Institute of Advanced Nuclear Systems, 2400 Mol, Belgium \\ 2 University of Liège, Institute of Physics, 4000 Liège, Belgium
}

\begin{abstract}
Recent extensions of the Liège intranuclear cascade (INCL) model concerning the mean field of the baryons, the pion sector and a refinement of the Pauli blocking for the first collision have led to an improved description of the production of radiotoxic isotopes induced by protons in thin targets. In this paper we have investigated the production of some highly radiotoxic isotopes in thick target. With this aim, the INCL4 model implemented in the MCNPX code has been modified. We have adapted the ALEPH code to perform the evolution of a spallation target. The standard INCL4 model and our modified INCL4 version were compared to the experimental results measured at PSI with a stack of lead and bismuth disks. The same conclusion drawn from thin target results is also obtained. In thin and in thick spallation targets, the production of the highly radiotoxic ${ }^{209} \mathrm{Po}$ and ${ }^{208}$ Po isotopes is reduced. In thick targets, the production of ${ }^{210} \mathrm{Po}$ and of ${ }^{210 \mathrm{~m}} \mathrm{Bi}$ are feeded by $(\mathrm{n}, \gamma)$ reactions and are not affected by the recent extensions.
\end{abstract}

\section{Introduction}

The assessment of the coupling between the particle accelerator and the sub-critical nuclear reactor core in acceleratordriven systems (ADS) requires the use of reliable high-energy collision models, to describe spallation reactions, embedded inside a general transport code to track the various emerging particles. Spallation reaction models are particularly important for matters such as neutron source distribution, heat generation, radiation damage and radiotoxicity.

Among the highest radiotoxic isotopes produced in leadbismuth spallation targets, we find isotopes of polonium $\left({ }^{210} \mathrm{Po},{ }^{209} \mathrm{Po}\right.$ and $\left.{ }^{208} \mathrm{Po}\right)$, of bismuth $\left({ }^{210 \mathrm{~m}} \mathrm{Bi},{ }^{204} \mathrm{Bi}\right)$ and of mercury $\left({ }^{194} \mathrm{Hg}\right)[1] .{ }^{210} \mathrm{Po}$ dominates the radiotoxicity initially and, ${ }^{209} \mathrm{Po}$ and ${ }^{208} \mathrm{Po}$, the one after 10 years. At equilibrium, the radiotoxicity comes mainly from ${ }^{210 \mathrm{~m}} \mathrm{Bi}$ and ${ }^{194} \mathrm{Hg}$.

The INCL4 model coupled to the ABLA evaporationfission model has been shown to give a fairly good description of a large amount of experimental measurements for protoninduced spallation reactions on thin targets in the $200 \mathrm{MeV}-$ $2 \mathrm{GeV}$ range [2]. This model is not yet perfect and some discrepancies remain: the production of polonium isotopes is quite overestimated. In the last years there have been new developments of the INCL4 cascade model, bearing on baryon mean field and pion dynamics $[3,4]$. The INCL4 model and these improvements will be briefly described in the section 2 .

Since these modifications led to a noticeable reduction of the cross sections involving few emitted nucleons and pions, they must have an effect on the production of residues close to the target. This improvement will be shown, in the section 3, by comparisons of the standard and the extended INCL4 model to reproduce the measurements obtained with thin target experiments. In section 4, we will investigate whether these extensions have an influence on the residue production inside a thick target. This validation will allow the study of the

\footnotetext{
${ }^{a}$ Presenting author, e-mail: taoust@sckcen.be
}

impact of spallation model improvements on particle transport inside media.

\section{The INCL4 model and improvements}

The INCL4 version is described in detail in the ref. [2] and, also including the recent improvements, in other contribution to this conference $[5,6]$. It is important to recall that the particle-nucleus collision is described by a Monte Carlo simulation. Initially the position and momentum of all target nucleon are randomly distributed in the nuclear volume and in a Fermi sphere, respectively. In the standard INCL4 version, a smooth initial density distribution is introduced, in concordance with electron scattering data. $N N \rightleftharpoons N N$, $N N \rightleftharpoons N \Delta$ and $\Delta \rightleftharpoons N \pi$ collisions are based on realistic parameterized cross sections and are subject to a consistent Pauli blocking. The cascade is stopped according to a criterion based on the time evolution of some physical quantities. The code accommodates nucleons and light composites as incident particles.

Among the recent extensions of the INCL4 model, the modifications considered in our assessment are:

- The isospin dependence of the mean field. To this aim, we introduce potential wells of different depths, $V_{0}^{p}$ and $V_{0}^{n}$ and different Fermi momenta for protons and neutrons. The depths are determined by requiring that the Fermi energies are equal to the respective separation energies [3].

- The well-known energy dependence of the mean field. Here a linear energy dependence is considered: $V_{0}^{i}(E)=$ $V_{0}^{i}\left(E_{F}^{i}\right)-\alpha_{i}\left(E-E_{F}^{i}\right)$ for $E<E_{0}$ and $V_{0}^{i}(E)=0$ for $E>E_{0}$. $E_{0}$ is the energy where the average potential becomes negligible and corresponds approximately to $200 \mathrm{MeV}$ of kinetic energy. The parameter $\alpha$ equals 0.23 (see ref. [3] for more details). 
- Better pion-nucleon cross sections at energy above the $\Delta$-resonance. These cross sections are parametrized from the Review of Particle Properties of ref. [7].

- Introduction of a mean field for the pions, determined from a global fit to a set of data bearing on $\pi$ production and $\pi$-nucleus cross sections [4]. From this study, for a lead nucleus, the (nuclear) pion mean field is close to $-16 \mathrm{MeV}$ for positive pions and close to $-45 \mathrm{MeV}$ for negative pions. An average Coulomb potential is added in the interior of the target nucleus and the usual Coulomb potential is introduced at the exterior.

- Refinement (hardening) of the Pauli blocking for the first nucleon-nucleon collision [8].

Overall these developments have an effect relatively weak on the neutron sector, except for the events where few collisions occur: the quasi-elastic peak and the high-energy neutron multiplicity are improved, and consequently the production of residues of masses close to the target mass are also improved.

\section{Thin target results}

Figure 1 shows excitation functions for the production of some residues whose mass is close to the target one, induced by protons impinging a ${ }^{209} \mathrm{Bi}$ nucleus. The modified INCL4

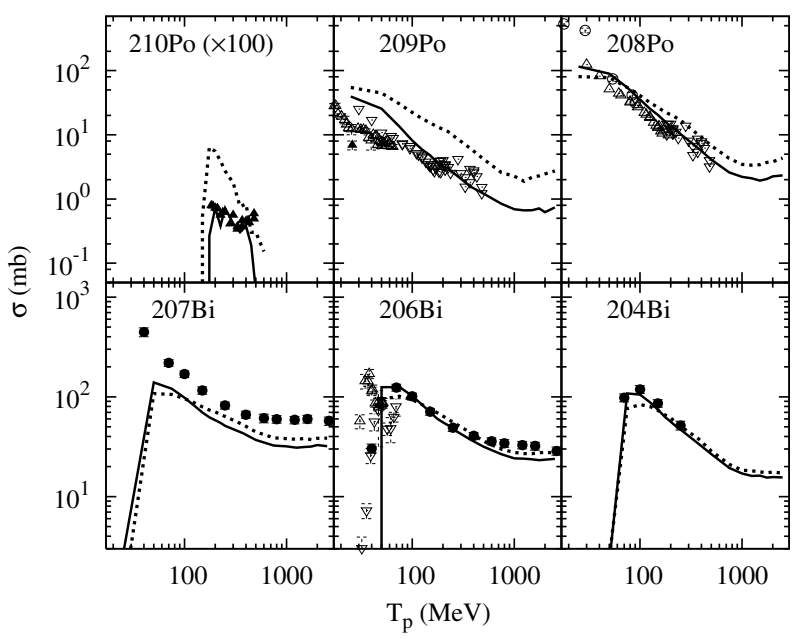

Fig. 1. Cross sections of production of residues, as functions of the kinetic energy of the incident proton impinging a ${ }^{209} \mathrm{Bi}$ nucleus. Results of the standard model and of the modified model are given by dotted lines and continuous lines, respectively. Experimental data (black points) comes from refs. [9-14].

model improves considerably the production of ${ }^{210} \mathrm{Po}$ and of ${ }^{209}$ Po.

The production of ${ }^{210} \mathrm{Po}$ is strongly reduced, mainly near to the threshold of the inelastic nucleon-nucleon cross section. This is linked to the reduction of the production of pions in the modified INCL4 model: the only way to produce ${ }^{210}$ Po from ${ }^{209} \mathrm{Bi}$ is the $\left(\mathrm{p}, \pi^{0}\right)$ reaction. This reduction is mostly induced by the introduction of the energy-dependence of the nucleon mean field which is negligible for proton of kinetic energy higher than $200 \mathrm{MeV}$.
The production of ${ }^{209} \mathrm{Po}$ and ${ }^{208} \mathrm{Po}$ is also reduced, but weakly. Here, mostly, this effect results from the decrease of the emission of cascade neutrons due to the implementation of the energy dependence of the nucleon mean field. The main way to produce ${ }^{209} \mathrm{Po}$ and ${ }^{208} \mathrm{Po}$ is from $(\mathrm{p}, \mathrm{n})$ reactions and from $(\mathrm{p}, 2 \mathrm{n})$ reactions, respectively. The production of isotopes of bismuth is less modified by our new implementations. When we stay away from isotopes close to the target, variations disappear, except for some rare earth.

\section{Thick target results}

In order to assess whether the conclusion drawn with thin target experiments are also observed with thick spallation targets, we have compared the predictions of the standard and the improved INCL4 models with experimental data for production of residual radio-nuclides, determined by $\gamma$-ray spectroscopy, in a stack of alternating $\mathrm{Pb}$ and $\mathrm{Bi}$ disks (30 in total) bombarded with $590 \mathrm{MeV}$ protons at PSI [15]. Each disk has a diameter of $10 \mathrm{~cm}$ and a thickness of $1 \mathrm{~cm}$. This stack was irradiated during $8.5 \mathrm{~m}$ and the $\gamma$-ray spectroscopy was performed some hours to some days after the irradiation.

To perform the transport of the particles inside this thick target we have used the MCNPX transport code, which contains the INCL4.2 model. Our modifications were also implemented (INCL4.4 model) and the MCNPX code was coupled to the ORIGEN evolution code through the use of the ALEPH system developed at SCK•CEN [16]. Since ALEPH is initially not conceived for high-energy particles, we have adapted it to spallation target evolution and we have used the HISTP option of MCNPX [17].

In figure 2 the theoretical estimates obtained in the first bismuth disk and for a time corresponding to the first campaign of measurements (after some hours) are confronted to the experimental results of ref. [19]. The way of measurement


Fig. 2. Isotopic density of $\mathrm{At}, \mathrm{Po}, \mathrm{Bi}$ and $\mathrm{Pb}$ produced in the second disk $\left({ }^{209} \mathrm{Bi}\right)$. Experimental data (black points) comes from ref. [19]. 
of the residues ( $\gamma$-ray spectroscopy) does not provide with experimental information concerning the highly radiotoxic isotopes created by spallation reactions, which are for most of them $\alpha$-emitters. Nevertheless the estimates of the INCL4 models (standard and modified) are close to the experimental values, except for all isomeric isotopes which are strongly underestimated. This results from the use of old data for the residual $\gamma$-deexcitation (PHTLIB data file) provided with MCNPX version 26a [18].

As observed from the thin target analysis, few differences appear between the two theoretical evaluations, except for the production of $Z=Z_{T}+1$ and $A=A_{T}-x$ with $x=0, \ldots, 3$ and for the production of $Z=Z_{T}+2$ isotopes, which are reduced (the subscript " $T$ " refers to the target). We have to note that for these isotopes the reduction is of the same magnitude for the thick and for the thin targets. For instance, the reduction of the production of ${ }^{209}$ Po from bismuth target is close to $60 \%$ in the figure 1 and the figure 2.

The production of $Z=Z_{T}+1$ and $A=A_{T}+1$ isotopes are not influenced by our implementations. In thick spallation targets, ${ }^{210 \mathrm{~m}} \mathrm{Bi}$ and ${ }^{210} \mathrm{Po}$ are mostly produced by $(\mathrm{n}, \gamma)$ reactions (followed by $\beta^{-}$-decay for the ${ }^{210} \mathrm{Po}$ ).

Only the first eleven disks were analysed in the ref. [19]. The calculated production of the most highly radiotoxic isotopes in these disks is given in figures 3 and 4 for the $\mathrm{Pb}$ disks and $\mathrm{Bi}$ disks, respectively. Because of the very long

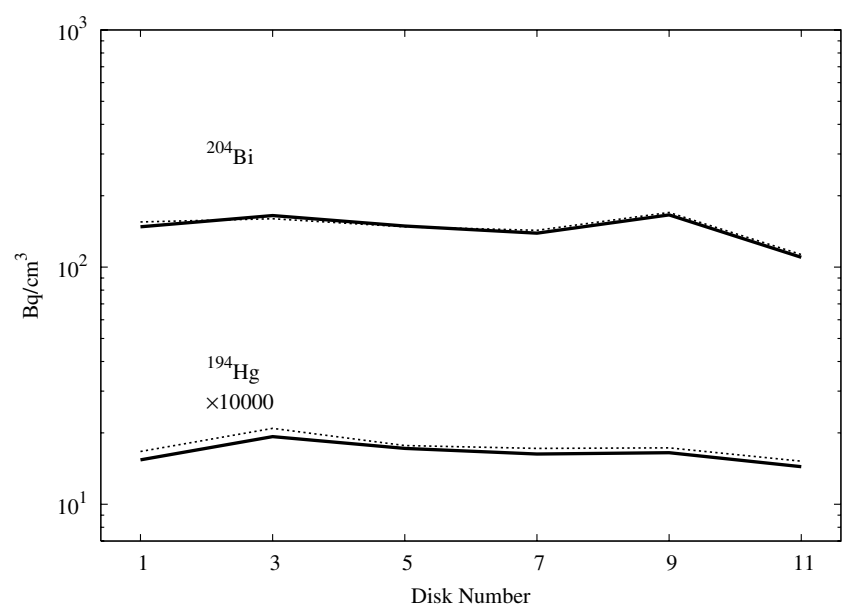

Fig. 3. Activity $\left(\mathrm{Bq} / \mathrm{cm}^{3}\right)$ of ${ }^{204} \mathrm{Bi}$ and ${ }^{194} \mathrm{Hg}$ induced in the $\mathrm{Pb}$ disks. Results of the standard model and the modified model are given by dotted lines and continuous lines, respectively.

half-life of the ${ }^{210 \mathrm{~m}} \mathrm{Bi}$, its activity is too weak and this isotope is not presented in figure 4. Except for the production of ${ }^{210} \mathrm{Po}$, which is feeded by the low energy neutron capture reaction, the production of the other isotopes remains quite constant in each disk. This shows that below the Bragg peak, spallation reactions are similar in each disk. The fact that the difference observed between the two INCL4 versions remains the same from disk to disk, seems normal since the emission of nucleons is weakly influenced by our modifications, which will be furthermore reduced by compensation effects.

The comparison of the figures 3 and 4 shows that the (weak) reduction of ${ }^{194} \mathrm{Hg}$ is more pronounced for the lead



Fig. 4. Activity $\left(\mathrm{Bq} / \mathrm{cm}^{3}\right)$ of ${ }^{204} \mathrm{Bi},{ }^{194} \mathrm{Hg},{ }^{208} \mathrm{Po},{ }^{209} \mathrm{Po}$ and ${ }^{210} \mathrm{Po}$ induced in the Bi disks. Same convention as in figure 3.

disk than for the bismuth ones. This could be also attributed to the fact that our modifications affect mainly the channels involving a small number of emissions of nucleons.

\section{Conclusion}

The improvement of the INCL4 model concerning the description of the nuclear mean field of the baryons and of the pions, combined to the extension of the pion-nucleon cross sections above the $\Delta$-resonance and to the refinement of the Pauli principle in the INCL4 model have allowed a better estimate of the production of polonium and of bismuth residues induced by protons in thin targets. The predicted ${ }^{210} \mathrm{Po}$ and ${ }^{209} \mathrm{Po}$ production cross sections are considerably reduced and are now very close to the experimental measurements.

The effect of our improvements for the production of some radiotoxic isotopes induced in thick target were performed for the stack of alternating $\mathrm{Pb}$ and $\mathrm{Bi}$ disks bombarded with $590 \mathrm{MeV}$ protons at PSI. The INCL4 model implemented in our version of the MCNPX code was updated and the evolution of this spallation target was performed by adapting the ALEPH code. For the few experimental measurements, the predictions of the standard and the modified INCL4 models remain close to each other and give satisfactory results. The use of old data for the residual $\gamma$-deexcitation in MCNPX26a gives a strong underestimation of the production of isomeric states. As observed from thin target analysis, the production of ${ }^{209} \mathrm{Po}$ and ${ }^{208}$ Po are strongly reduced. Comparing the effects of our modifications in thin targets and in thick targets, the observed variations remain similar. The production of ${ }^{210} \mathrm{Po}$ and of ${ }^{210 \mathrm{~m}} \mathrm{Bi}$ in the bismuth disks are not influenced by our new implementations, since these isotopes are mainly produced by $(\mathrm{n}, \gamma)$ reactions, followed, for the ${ }^{210} \mathrm{Po}$, by $\beta^{-}$decay.

From the point of view of radiotoxicity, it seems that a lot of the highly radiotoxic isotopes are produced on bismuth by spallation reaction. However, we have to underline that the thick spallation target used here is not surrounded by a sub-critical core and the reaction rate of neutron capture is here much lower than in an Accelerator-Driven System. 
This work has been partially done in the frame of the EU IP EUROTRANS project (European Union Contract No. FI6W-CT2004-516520). We acknowledge the EU finacial support.

\section{References}

1. H. Trellue, in Proceedings of the Neutrons for Science (NFS), SPIRAL2 Workshop, GANIL, Caen, France, Dec. 2004.

2. A. Boudard, J. Cugnon, S. Leray, C. Volant, Phys. Rev. C 66, 044615 (2002).

3. Th. Aoust, J. Cugnon, Eur. Phys. J. A 21, 79 (2004).

4. Th. Aoust, J. Cugnon, Phys. Rev. C 74, 064607 (2006).

5. A. Boudard et al. (these proceedings).

6. J. Cugnon et al. (these proceedings).

7. H. Hagiwara et al., Phys. Rev. D 66, 010001 (2002).

8. J. Cugnon, P. Henrotte, Eur. Phys. J. A 16, 393 (2003).
9. Y.E. Titarenko et al., Experimental and theoretical studies of the yields of residual product nuclei produced in thin $\mathrm{Pb}$ and $\mathrm{Bi}$ targets irradiated by 40-2600 MeV, Final Technical Report on the ISTC Project No. 2002, 2004.

10. T.E. Ward et al., Phys. Rev. C 24, 588 (1998).

11. J.M. Auria et al., Phys. Rev. C 30, 236 (1984).

12. K. Miyano et al., Nucl. Phys. A 230, 98 (1974).

13. Y.L. Beyek et al., Nucl. Phys. A 99, 131 (1967).

14. P.J. Daly et al., Nucl. Phys. 56, 322 (1964).

15. K. van der Meer et al., Nucl. Instrum. Meth. B 217, 202 (2004).

16. W. Haeck, B. Verboomen, Nucl. Sci. Eng. 156, 1 (2007).

17. Th. Aoust, J. Cugnon, W. Haeck, in Proceedings of the 12th International Conference on Emerging Nuclear Energy Systems (ICENES05), Bruxelles, Belgium, August 21-26, 2005.

18. J.S. Hendricks et al., MCNPX, Version 26b, Los Alamos Report, LA-UR-06-3248, 2006.

19. C. Dams, End Study thesis, XIOS Hogeschool Limbourg, 2005. 